\title{
Carnets
}

Revue électronique d'études françaises de l'APEF

Deuxième série - 13 | 2018

Corps, rythmes et voix : en/jeux littéraires et artistiques

\section{Les voix du conteur entre poéticité et ironie dans \\ Le Coq de bruyère, Le Médianoche amoureux et d'autres récits de Michel Tournier}

\section{Annie Urbanik-Rizk}

\section{(2) OpenEdition}

\section{Journals}

Édition électronique

URL : http://journals.openedition.org/carnets/2593

DOI : $10.4000 /$ carnets.2593

ISSN : 1646-7698

Éditeur

APEF

Référence électronique

Annie Urbanik-Rizk, "Les voix du conteur entre poéticité et ironie dans Le Coq de bruyère, Le

Médianoche amoureux et d'autres récits de Michel Tournier », Carnets [En ligne], Deuxième série -

13 | 2018, mis en ligne le 31 mai 2018, consulté le 20 avril 2019. URL : http://journals.openedition.org/ carnets/2593; DOI : 10.4000/carnets.2593

Ce document a été généré automatiquement le 20 avril 2019.

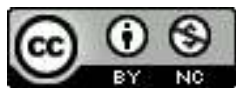

Carnets est mis à disposition selon les termes de la licence Creative Commons - Atribution - Pas d'utilisation commerciale 4.0 International. 


\title{
Les voix du conteur entre poéticité et ironie dans Le Coq de bruyère, Le Médianoche amoureux et d'autres récits de Michel Tournier
}

\author{
Annie Urbanik-Rizk
}

1 Michel Tournier nous laisse une œuvre incroyablement diverse, fantaisiste et dont la forte empreinte se perpétue durablement dans l'imaginaire des lecteurs. La raison n'en est pas seulement le caractère mythologique sous l'apparence du quotidien, ni l'extraordinaire inventivité. Il laisse la mémoire d'une voix, audible à travers l'écrit, forte et simple comme celle du récitant au théâtre ou du griot africain. Les textes littéraires dont il revendique l'influence capitale sont pour la plupart des lectures d'enfance, par exemple La Reine des neiges d'Andersen (Andersen, 2005 [1844]) ou Le merveilleux voyage de Nils Holgersson de Selma Lagerlöf (Lagerlöf, 1906), dont le charme repose sur la transfiguration du réel par l'entremise du conte. Cette vision magique des choses est permise essentiellement grâce à la vertu de l'oralité et au retour à l'aptitude poétique de l'enfance. On peut comprendre pourquoi Michel Tournier aimait à soumettre ses récits en cours d'écriture à des groupes d'enfants, parfois ses simples voisins, plus exigeants que toute académie. On dénombre une part importante de récits courts dans sa production et qui se sont orientés, au fil des années, vers une sobriété de plus en plus importante, quand parfois certains comme la robinsonnade Vendredi ou les limbes du Pacifique (Tournier, 1967) ont aussi donné lieu à une deuxième version adressée au public enfantin Vendredi ou la vie sauvage (Tournier, 1971). Oralité, enfance, goût pour les sortilèges qui émanent de toute apparence sont bien trois aspects d'une exigence poétique... pourtant paradoxale.

2 La distance critique, l'ironie, le sourire bienveillant sont toujours lovés dans une prose qui n'est pas aussi simple qu'elle ne parait, sous un récit qui comporte une signification cryptée et qui met de la métaphysique dans une miche de pain. L'objet de cette réflexion sera de déceler la part de sérieux dans le sourire sarcastique, l'élaboration d'un sens sous 
la critique néantisante et la repoétisation qui naît de la réécriture de certains contes ou de certains mythes.

Dans le Coq de bruyère ou Les Sept contes (Tournier, 1984) dans la version pour enfants, l'écrivain s'amuse librement, dans une forme extrêmement brève parfois, («La Mère Noël » fait deux pages et demie) à reprendre des contes folkloriques en les grimant, voire en les dénaturant complètement. Le Petit Poucet refusant de vivre dans une tour moderne fait une fugue en compagnie de lapins et une institutrice laïque allaite le petit Jésus dans une crèche vivante. Le charme de la voix du conteur se double d'un sourire amusé et complice. Dans le Médianoche amoureux (Tournier, 1989), Tournier écrit un recueil de nouvelles à la manière du Décaméron de Bocacce (Boccace, 2006 [1349-1353]) ou de l'Heptaméron de Marguerite de Navarre (Marguerite de Navarre, 1559), puisque chacun des convives du repas de minuit doit raconter une histoire sur le thème du double ou de la répétition, afin de sauver de la grisaille du quotidien un couple en perdition. Multiples voix enchâssées dans une voix, ces récits subtils qui se terminent bien mettent en scène les pouvoirs magiques du conte. Quant à Eléazar ou la source et le buisson (Tournier, 1996) dont le format est intermédiaire entre la nouvelle et le roman, il réécrit l'odyssée de Moïse en direction de la Terre Promise, par le biais d'un migrant en quête de la Californie et s'apparente à la même démarche.

4 Nous verrons donc comment à travers ces formes multiples et mouvantes, Michel Tournier met en scène le charme premier du conte, puis comment il semble en briser la poésie première par l'ironie, enfin qu'une repoétisation s'accomplit, qui, offre de surcroît un sens lisible aux lecteurs.

\section{La voix du conteur et le charme premier du conte}

\section{A. Des formules rituelles}

5 Comme le supposent tous les récits folkloriques ritualisés en des moments ou des lieux spécifiques, le soir à la veillée, sous un arbre à palabres en Afrique, au moment du coucher pour les tout-petits, des formules canoniques marquent les étapes charnières. L'embrayeur fondamental du conte, le «il était une fois", à force de répétition conventionnelle a perdu en signification ce qu'il a gagné en magie. Il ne sert plus simplement à indiquer une rupture avec le temps présent ou avec la réalité, mais à glisser insensiblement vers l'impersonnalité ensorceleuse du récit légendaire. Le «carmen » au sens latin, le charme de la voix humaine agit par cette formule, censée interpeler le lecteur transformé en auditeur. Cette poésie d'un temps immémorial initie le tout premier conte du Coq de bruyère (Tournier, 1984 ) intitulé «La famille Adam », où il est justement question des commencements bibliques : «Au commencement il n'y avait sur la terre ni herbe ni arbre. Partout s'étendait un vaste désert de poussière et de cailloux. » $(\mathrm{CB}, 11)$.

6 La formule adverbiale, in illo tempore, associée à un usage poétique de l'imparfait, qui marque la suspension temporelle d'une époque située en dehors de toute chronologie invite à l'écoute attentive, ainsi qu'à une forme de présence quasi-charnelle. Locuteur et auditeur sont rassemblés dans une pratique cérémonielle. Le "ce soir-là » par lequel débute « La fugue du Petit Poucet » (p. 49) joue à peu près le même rôle, même si le soustitre de l'histoire «conte de Noël» nous indique déjà la couleur. Quant au récit d'Amandine ou les deux jardins, il est qualifié de « conte initiatique ». Passage d'un état 
d'ignorance ou d'innocence à celui de connaissance ou d'expérience qui concerne le personnage ou bien le lecteur, le sous-titre ne le dit pas... Le mystère est la tonalité majeure de la voix du conteur.

\section{B. L'oralité}

7 Outre le fait de signaler l'entrée dans le royaume du conte par un certain nombre d'indicateurs codés, une oralité propre à Michel Tournier transparaît dans tous ses récits, quels qu'ils soient. Toujours dans «la famille Adam», l'auteur interpelle le lecteur en adoptant un ton didactique et en employant des interrogatives directes: "A quoi ressemblait le premier homme ? Il ressemblait à Jehovah qui l'avait créé à son image. » ( $C B, 11)$.

Un peu plus loin :

Il faut savoir que les choses ont commencé de la sorte pour comprendre la suite.

Adam et Ève, on le sait, furent chassés du Paradis par Jéhovah.

Naturellement, cette chute hors du Paradis ne représentait pas du tout la même chose pour Adam et pour Ève. (CB, 14)

9 Ce type de modalisateur adverbial instaure sous les dehors de l'insistance, une forme de complicité entre l'auteur/récitant et le lecteur en chair et en os aux antipodes de ce qu'Umberto Eco nomme « le lecteur modèle » (Eco, 1979), abstrait et formel.

10 Dans La Fugue du petit Poucet, lorsque Pierre s'échappe de la cabane familiale pour éviter un déménagement dans une grande ville aseptisée, il est pris en autostop par un camionneur qui le dépose en pleine nuit sur le bord de la route. Tournier ménage ses effets en faisant rebondir l'action par le biais de questions et de réponses censées relancer l'intérêt, un peu comme au guignol: "Des étoiles dansent autour de lui avec des exclamations et des rires argentins. Des étoiles? Non, des lanternes. Ce sont des gnomes qui les tiennent. Des gnomes? Non, des petites filles. Elles se pressent autour de Pierre.» $(C B, 55)$.

11 La simplicité de ces questions courtes contribue au mystère. Sept petites filles apparaîtront en effet surgies de nulle part, sinon des souvenirs du conte initial de Perrault. La voix de Tournier redouble, transforme et fait surgir à la fois le conte initial et sa métamorphose. Tout en étant nées de l'arbitraire de l'écrivain, ces apparitions trouvent leur place et sont motivées par une forme de reconnaissance et d'attente du public, en communion avec le charme du récit.

12 Dans des récits plus longs comme Eléazar ou la source et le buisson (Tournier, $1998^{1}$ ) pourtant plus proches de l'écriture du roman, on ne peut qu'être frappé par le rythme des phrases toutes prêtes à être oralisées, comme le soulignait à juste titre Jean-Bernard Vray. (Jean-Bernard Vray, 1998, 11-12). Les sentences qui parsèment le récit y ont la rythmique scandée des proverbes: "L'enfant et le vieillard/ flottent sans attaches/à la surface de l'existence » ( $E, 11$ et 12$)$ et la vie pastorale d'Eleazar est narrée avec l'ampleur du verset biblique: «les travaux et les jours d'un berger n'annoncent pas évidemment ceux d'un pasteur d'hommes. » $(E, 15)$

\section{Un univers didactique}

13 A cela vient s'ajouter la présence de sentences, de stéréotypes et de clichés qui viennent ponctuer les événements du récit, comme autant de morales d'un vaste apologue. La voix 
de Tournier s'oriente donc autant du côté du charme de la narration que de la sagesse du fabuliste, avec ses personnages animaliers, son onomastique symbolique et sa signification transparente pour un public enfantin. Dans La Fugue du petit Poucet, la leçon morale est portée par le père des sept petites filles. Il s'appelle "Logre », nom imaginé comme dérivation comique du personnage effrayant du conte d'origine. Le signifiant connotant l'effroi de la dévoration se métamorphose en son inverse rassurant. Logre n'est qu'un hippie bienveillant qui distribue des cigarettes apaisantes à toute sa famille et joue de la guitare. Il est le double du poète puisqu'il raconte à sa manière l'histoire des origines en transmettant une morale d'harmonie avec la nature primitive, en accord avec les intuitions de Pierre :

Qu'est-ce qu'un arbre? [...] L'arbre ne fait qu'un avec le vent et le soleil. Il tète directement sa vie à ces deux mamelles du cosmos, vent et soleil. Il n'est que cette attente. Il n'est qu'un immense réseau de feuilles tendu dans l'attente du vent et du soleil. (CB, 54)

La description poétique du paradis terrestre dont fut chassé Adam est transposée en une simple description nostalgique. Pierre Poucet va perdre, en s'installant à la ville, son paradis campagnard; c'est une espèce de Chute. Mais immédiatement après ce passage lyrique, la police vient interrompre le beau discours du hippie et emmène Logre qu'elle accuse de trafic de drogue et de détournement de mineur. Les exemples de cette transition brutale entre l'idéalité et la brutalité du réel sont innombrables dans les récits de Tournier, et d'autant plus efficaces qu'ils sont brefs. La collusion du poétique et du trivial, le passage d'un registre à l'autre est la marque de fabrique d'un écrivain qui déstabilise le lecteur autant qu'il le ravit. Le charme est en quelque sorte rompu.

Quelles sont la place et le sens de cette rupture du poétique, du retour en force du réel, en un mot de l'ironie dans le récit?

\section{L'ironie de l'écrivain et la rupture du poétique}

\section{A. Réécriture, inversion, déplacements}

Force est de constater que l'écriture de Tournier n'est pas poétique la majeure partie du temps et qu'il refuse même ce genre canonique dont il récuse les créations formelles complexes, préférant la simplicité du style. Tout se passe comme si l'auteur, dans sa révolte contre les institutions littéraires, voulait simultanément brouiller tous les genres et à sa manière, revenir aux éléments les plus archaïques de la littérature, ceux qui ont précédé l'écrit, ceux qu'analyse Lévi-Strauss dans Anthropologie structurale II. (Lévi-Strauss, 1973). De là vient ce principe de réécriture critique adossé au retour du plus fondamental : le plaisir de la narration. La seule observation des titres des contes du Coq de bruyère en dit long sur le côté systématique de la réécriture qui concerne aussi bien les contes folkloriques (le petit Poucet dans «La fugue du Petit Poucet», le conte de Noël dans « la Mère Noël ») que les mythes (la Genèse dans « la famille Adam », Robinson dans «La fin de Robinson Crusoë», l'iconographie chrétienne dans «Les suaires de Véronique »).

Observons dans cet esprit le traitement du mythe de l'androgyne emprunté à Platon, croisé avec la légende biblique de la création d'Eve. Le palimpseste est clair: Tournier superpose deux histoires sans effacer totalement la première. La simplification, l'usage d'une langue familière quasi-infantile, l'humour qui dépeint la surprise d'Adam laisse 
supposer une seconde voix amusée ainsi qu'un sourire narquois, qui semble ôter au mythe de son sérieux.

- Maintenant, dit Jéhovah, je vais te couper en deux. Dors!

- Me couper en deux ! s'exclama Adam.

Mais son rire s'éteignit bientôt et il tomba endormi. Alors, Jehovah retira de son corps tout ce qui était femme : les seins, le petit trou, la matrice.

Et ces morceaux, il les mit dans un autre homme qu'il modela à côté dans la terre humide et grasse du Paradis.

Et il appela cet autre homme : femme.

Lorsqu' Adam se réveilla, il sauta sur ses pieds et faillit s'envoler tant il se sentit léger. $(C B, 13)$

Le mythe de l'androgyne est une métaphore personnelle de l'écrivain qui figure métaphoriquement à la fois le mystère de la sexualité, née d'une damnation divine ainsi que le double et la gémellité. Belle coalescence de deux agglomérats de signifiants qui déplace et superpose deux ensembles de légendes.

Un peu plus loin, le mythe de Caïn et Abel est revisité selon un principe d'irrévérence simplificatrice.

Le premier, Caïn, était tout le portrait de sa mère : blond, dodu, calme et très porté à dormir. [...] Tout autre était son jeune frère, Abel. Celui-là, c'était tout le portrait de son coureur de père. Jéhovah trouvait de l'insolence et de la rébellion dans cet Eden II que Caïn avait fait sortir du sol ingrat du désert. $(C B, 15)$

La rivalité des deux frères aboutissant au meurtre est relue à partir des catégories opposées du nomade et du sédentaire, dont l'une est la préférée de Dieu. Subtile lecture rétrécissante et blasphématoire pour certains $-\mathrm{du}$ meurtre primitif fondateur de la civilisation.

\section{B. L'ironie}

21 Le procédé de l'ironie qui consiste à suggérer un sens différent de l'énoncé, rend hommage au texte initial tout en extirpant sa solennité. En quelque sorte, il le détruit. L'ironie confine au burlesque qui désacralise les idoles. Il y a du vieillard Anchise ridiculisé par Scaron dans son Virgile travesti dans le portrait que fait Tournier de Jehovah :

C'était Jehovah, fatigué, éreinté, fourbu par la vie nomade qu'il menait depuis tant d'années avec les fils d'Abel, cahoté à dos d'homme dans une Arche d'Alliance vermoulue, qui puait le suint de bélier. (CB, 17)

La familiarisation, sur laquelle nous reviendrons participe au principe de rétrécissement de la légende au niveau du réel, voire du quotidien. L'incarnation divine se transforme en histoire de famille « Le petit-fils (Caïn, fils d'Adam, créé par Dieu) serra le grand-père sur son cœur » $(C B, 17)$

Dans certains cas, l'histoire commence d'emblée par la rupture du charme poétique du conte de référence. Ainsi, dans « La fugue du petit Poucet ", le récit commence in medias res, par une annonce du père de famille à sa femme et à ses enfants.

Ce soir-là, le commandant Poucet paraissait décidé à en finir avec les airs mystérieux qu'il prenait depuis plusieurs semaines, et à dévoiler ses batteries.

- Eh bien voilà, dit-il au dessert après un silence de recueillement. On déménage. Bièvres, le pavillon de traviole, le bout de jardin avec nos dix salades et nos trois lapins, c'est terminé ! $(C B, 49)$ 
L'ironie à l'œuvre ici dans sa bienveillance familière, correspond à la définition nuancée qu'en donne Jankélévitch dans son essai L'Ironie :

Qu'est-ce que l'ironie sinon la conscience, mais une bonne conscience joyeuse-ce en quoi elle se distingue de l'hypocrisie? Pas d'humour sans amour ni d'ironie sans joie. L'ironie en somme sauve ce qui peut être sauvé. (Jankélévitch, 1936 : 57)

Cet humour bienveillant est aussi présent dans les nouvelles plus longues du Médianoche amoureux (Tournier, 1989)². Dans «Lucie ou la femme sans ombre », l'ironie s'exerce dans l'onomastique, les jeux de mots, une sorte de présence affectueuse du narrateur. Évoquant son amour pour son institutrice, le narrateur épilogue sur l'ambiguïté du terme " maîtresse ", origine de la fantaisie de cette histoire. " Il est en effet remarquable que le français emploie le même mot pour désigner l'amante d'un homme marié, sa seconde femme en somme, et l'enseignant qui se charge des écoliers les plus jeunes. » (Tournier, 1989 : 69) Les enfants de Lucie s'appellent Tibo et Tijoli, onomastique ridiculement simplificatrice. Rapprocher le lointain, rendre familier l'étrange et rassurant l'inquiétant, tels sont les effets de cette seconde voix ironique du narrateur aux antipodes d'un travail de sape et de dépoétisation.

\section{Procédés de familiarisation}

Ce n'est pas seulement la bienveillance de l'émetteur qui rend au récit son rythme allègre, et corps à la présence souriante de l'écrivain. Le destinataire en chair et en os est également rendu présent par les très nombreux procédés de familiarisation. Le plus célèbre, car sans doute le plus efficace se trouve dans la nouvelle « Pierrot ou les secrets de la nuit. » Le village de Pouldreuzic apparaît, net, simplifié comme sur une image d'Epinal, d'une simplicité enfantine dans sa binarité.

Deux petites maisons blanches se faisaient face dans le village de Pouldreuzic. L'une était la blanchisserie. Personne ne se souvenait du vrai nom de la blanchisseuse, car tout le monde l'appelait Colombine en raison de sa robe neigeuse qui la faisait ressembler à une colombe. L'autre était la maison de Pierrot. (MA, 122)

Cette histoire de trahison et de séduction oppose les contraires : la blanche Colombine sera attirée par les couleurs d'Arlequin, peintre en bâtiment. A la fin du conte les deux rivaux se réconcilient et communient en mangeant la brioche en forme de Colombine, " réconciliation de l'âme solaire de Colombine avec la partie nocturne et profonde d'ellemême qui l'effrayait tout d'abord. » (Bouloumié, 1988 : 129).

Mais la trajectoire du récit va en réalité de la familiarité au symbole philosophique. Les jeux d'opposition sont expliqués à la première page par des raisons pratiques :

Pierrot et Colombine avaient grandi ensemble sur les bancs de l'école du village. Ils étaient si souvent réunis que tout le monde imaginait que plus tard, ils se marieraient. Pourtant, la vie les avait séparés lorsque Pierrot était devenu mitron et Colombine blanchisseuse. Forcément, un mitron travaille la nuit, afin que tout le village ait du pain frais et des croissants chauds le matin. Une blanchisseuse travaille le jour pour étendre son linge au soleil. (MA, 258)

Pour Arlette Bouloumié, les deux personnages de Pierrot et d'Arlequin répondent à une logique gémellaire, à une dualité symbolique.

Pierrot et Arlequin s'opposent comme les deux moitiés complémentaires de l'âme dont tantôt l'une, tantôt l'autre domine. » [...] « Pierrot s'oppose à Arlequin comme le blanc et le noir à la couleur, comme la nuit au jour, comme la nuit au soleil, comme ce qui est bas - Pierrot travaille dans une cave - à ce qui est haut- 
Arlequin est sur son perchoir »

"La dualité est au cœur de ce conte au sens secret et philosophique. »

«L'éloge de Pierrot, c'est la profondeur. Arlequin est brillant mais superficiel.

(Bouloumié, 1988, 129) êtres qui transfigure le quotidien. Cette véritable épiphanie du réel, au sens de James Joyce se lit à la fois dans le détail et dans le souffle quasi-surréaliste de celui qui voit une réalité supérieure derrière tout élément du réel. Reprenons le conte « La Fugue du petit Poucet » qui est un modèle de familiarisation et de transposition d'une histoire à la société contemporaine. Le seul regard de l'enfant échappé de sa prison familiale et qui découvre le monde naïvement métamorphose poétiquement le réel : «C'est la première fois que Pierre voyage dans un poids lourd. Comme on est haut perché ! On se croirait sur le dos d'un éléphant. » ou plus loin, dans la frayeur de la solitude au milieu de la nuit, les petits lapins qu'il a emmenés lui servent de réconfort :

Tiens, on va sortir les lapins. Ça tient chaud des lapins vivants. Ça remplace une couverture. C'est une couverture vivante. Ils se massent contre Pierre en enfonçant leur petit museau dans ses vêtements. Je suis leur terrier, pense-t-il en souriant. Un terrier vivant. $(C B, 51)$ du réel. Ce récit est pourtant inscrit dans la trivialité de la nouvelle et narre des faits violents puisqu'il raconte un viol et un parricide. L'incipit évoque la fraîcheur et la beauté du printemps favorable aux amants et à l'expression de la fine-amor.

Parce qu'il est fragile et tardif, le printemps des Hautes Terres d'Ecosse possède pour les hommes et les femmes de ce pays un charme d'une douceur exquise. Ils guettent avec une impatience enfantine le retour des vanneaux dans le ciel tourmenté, le cri amoureux des grouses des marais et les premières taches mauves des crocus sur l'herbe rare des collines. (MA, 226)

Tout en reprenant le topos du printemps propre au récit médiéval, Tournier construit une poétique du récit autonome en cherchant à restaurer son enchantement. Il convoque une réalité lointaine, celle des cours du pays d'Oc afin de créer un contraste extrêmement violent avec la brutalité des événements, le viol de la douce Colombelle par le géant Tiphaine. Au moment de la vengeance dictée par un père défunt, le héros épique, issu de cet amour contre-nature est métamorphosé «Jacques, nimbé de lumière, n'obéissait plus, n'entendait plus rien, comme porté par la force irrésistible de son destin. [...] l'enfant blond, bleu et rose, vêtu de soie et de tartan, avait l'éclat irréel d'une apparition. (MA, 246) 
Ayant repris de façon très libre les vers de La Légende des siècles, L'Aigle du casque de Victor Hugo, Michel Tournier rend hommage au grand poète tout en s'excusant de son extrapolation : il a inventé la nécessité pour Jacques de venger la mort de ses parents, inexpliquée de Victor Hugo. Cet ajout transfigure le personnage de l'enfant bâtard en élu de Dieu, audace qui demeure malgré tout cohérente avec le merveilleux chrétien du récit médiéval.

\section{B. Une poétique tourniérienne de la simplicité, une vision binaire des choses}

Derrière ces fantaisies constantes, ces distorsions parfois outrancières des récits de base, Tournier n'use pas du palimpseste par simple goût de l'amusement ou de la plaisanterie. Son propos vise souvent une simplification binaire qui participe à la fois d'un souci didactique et d'une vision ontologique. Il serait aisé de reprendre chacun des exemples mentionnés pour leur poésie du quotidien ou au contraire leurs effets de familiarisation prosaïque et d'en montrer la portée philosophique. Ainsi la fin d'Eléazar ou la source et le buisson explicite-t-elle le titre tout en s'appuyant sur des éléments bibliques. Le pasteur Eléazar, comme Moïse qui ne put arriver en terre d'Israël, meurt avant d'arriver en Californie :

La Source et le Buisson, dit-il d'une voix plus forte. Il faut choisir entre cette eau chantante qui jaillit à nos pieds et descend vers la vallée, et le buisson ardent dont la flamme monte du désert vers le ciel. Josué, fils de Nun, de la tribu d'Ephraïm, tu choisiras la source. Je te confie mon peuple? Esther, Benjamin et Cora, pour que tu les conduises sains et saufs en Terre promise. Sois juste et miséricordieux. $(E, 139)$

Une voix repoétise ce récit dont la toute dernière ligne est burlesque avec la mention du petit âne de cirque, mais il s'agit de la voix du personnage qui s'exprime comme un prophète.

La «fugue du petit Poucet» oppose deux visions du monde, symbolisées par les contraintes de la modernité urbaine aseptisée d'un côté et par la liberté au sein de la nature de l'autre. La dernière page nous montre un enfant libéré du carcan moderne grâce aux bottes offertes par le hippie Logre. L'hérö̈ne d'Amandine ou les deux jardins se libère aussi d'un univers tout aussi rassurant qu'étouffant en suivant un chat qui la mène vers le jardin luxuriant et le désordre de la maison voisine. Presque tous les contes font l'apologie d'une libération en termes d'initiation. Le récit le plus court, « La Mère Noël », s'ouvre sur une opposition idéologique cette fois, celle qui oppose les croyants et le village laïque. A la fin, ces contraires sont réconciliés par une image mariale: l'institutrice donne le sein à son bébé, qui sert de figurant pour la crèche vivante de l'église. Dans "Lucie ou la femme sans ombre", conte lui-même dédoublé, le titre annonce la dualité qui oppose l'ombre et la lumière, suggéré par le nom de Lucie. La nuit où elle recueille le narrateur dans la chaleur de son lit est une métaphore de l'initiation, parenthèse enchantée dans un univers répressif. Tout comme Bettelheim dans La Psychanalyse des contes de fées, (Bettelheim, 1976) Tournier apporte au conte une valeur de catharsis pour un lecteur enfant ou adolescent, à même de résoudre en imagination ses propres contradictions par la magie simplifiante de la narration. 


\section{Un sens-poético-philosophique}

40 Mais ces oppositions théorisées dans les essais Le Miroir des idées ou encore Le pied de la lettre ne nuisent-elles pas par leur abstraction et leur portée philosophique à ce charme premier que nous avons signalé dans un premier temps? Comment la voix du conteur, qu'elle soit première sous la forme du récitant, secondarisée dans une bienveillant ironie, ou intra-diégétique par l'entremise d'un personnage à la parole forte, comment cette voix peut-elle encore avoir une musicalité, un rythme, un corps dès lors que des catégories conceptuelles sont à l'œuvre ? Dans quelle mesure peut-on parler de dimension " poéticophilosophique?»

41 Le conte «le Nain rouge » nous offre sans doute des éléments de réponse. Il est l'un de ceux que l'auteur a le plus souvent cités pour démontrer que le conte permet d'offrir des réponses à des questions philosophiques. Dans ce récit, selon lui la quantité et la qualité se voient évaluées respectivement. Deux métamorphoses servent de vérités expérimentales au personnage du nain. Il devient l'amant surpuissant d'Edith, une femme en instance de divorce, après s'être dénudé et avoir passé le peignoir de son époux, disqualifié sur ce plan. «Edith, qui ne divorçait qu'en raison de l'insuffisance de son trop beau mari - » (Tournier, $1984: 107)$

42 Ensuite, lorsqu'il devient nain de cirque, il organise des séances destinées aux seuls enfants afin de perdre pour une fois son infériorité physique face aux spectateurs. Supériorité de la qualité sur la quantité dans un premier temps, relativité dans un second temps sont les vérités énoncées, dans un processus où les rebondissements s'accompagnent de beaucoup d'humour.

43 La nouvelle Pierrot ou les secrets de la nuit concilie davantage le poétique et le philosophique, alors que le conte précédent était plutôt une alliance de l'humour et de la philosophie. Dans un article du Monde du 21 décembre 1979, voici ce que dit l'auteur :

A travers les deux petites poupées du théâtre italien, ce sont deux visions du monde qui s'affrontent: la substance contre la surface, la matière contre la forme, l'essence contre l'accident. De grands échos retentissent dans ces puérils porteparole. C'est Gœthe et Newton séparés sur la théorie des couleurs, c'est Parménide contre Héraclite. (Tournier, 1979)

Le contraste entre un monde coloré et un univers en noir et blanc se récite en termes de rivalité amoureuse où le noir et blanc sort vainqueur, la couleur étant associée à la superficialité.

La prééminence de la blancheur est annoncée d'emblée aux premières lignes en une formule poétique où l'onomastique, le primat de l'image et l'imparfait jouent pleinement leur rôle : « Personne ne se souvenait du vrai nom de la blanchisseuse, car tout le monde l'appelait Colombine, en raison de sa robe neigeuse qui la faisait ressembler à une colombe. » (MA 258). Quant à Arlequin, malgré sa multitude de couleurs, il est présenté en termes privatifs, qui forment une prolepse :

L'homme vif, souple, aux joues vermeilles, aux cheveux roux et frisés, était vêtu d'une sorte de collant composé d'une mosaïque de petits losanges bariolés. Il y avait là toutes les couleurs de l'arc-en-ciel, plus quelques autres encore, mais aucun losange n'était blanc ni noir. (MA134)

Magnifique paradoxe et retournement sur lequel nous pouvons conclure à la subtilité de la parole de Tournier chez qui l'apparence est aussi trompeuse que le manteau 
d'Arlequin. L'essentiel demeure la voix définie ainsi dans "Tristan vox » : "Pour rester sans visage ni regard, les voix n'en avaient que plus de mystère, et leur magie agissait avec une efficacité parfois redoutable sur les hommes et les femmes à l'écoute. » $(M A, 57)$

Pour conclure, il importe de souligner qu'en première analyse, les récits de Tournier parce qu'ils sont souvent troublants et jouent avec la plus ordinaire des réalités, ne semblent pas mettre en œuvre la voix habituelle des conteurs de fables.

Néanmoins, au-delà de la diversité des genres et des catégories dans lesquelles l'auteur incorpore ses récits- conte, nouvelles, romans, essais, dialogues- il demeure toujours une oralité singulière, faite avant tout du désir de séduire le lecteur réel au plus intime de son esprit d'enfance. L'attention portée aux effets produits conduit parfois à briser, déplacer, contester ce qui constitue le charme habituel d'une voix de conteur. «Comment expliquer la magie de cette voix ?» [...] « elle avait [...] l'immense privilège de s'adresser aux yeux de l'âme et non à ceux du corps. » $(M A, 126)$

Loin de détruire les qualités de suspense de la narration, d'en assécher les images ou de sombrer dans une triste trivialité, Michel Tournier parvient à transfigurer le quotidien en une épiphanie propre à celui qui sait voir le merveilleux sous le plus humble détail.

Ainsi est-il possible de parler de re-poétisation et de renouvellement multiforme de la fiction par la magie d'un regard où tout devient mythologique grâce à la pluralité des voix, «âmes qui s'envolent à tire-d'aile vers des milliers d'autres âmes. » (MA, 141) «Tristan vox ». Les voix, pour Tournier, ont cette triple qualité d'être simultanément, corps, esprit et âme.

\section{BIBLIOGRAPHIE}

ANDERSEN, Hans Christian, (2005). Contes, traduction de Marc Auchet, Paris, Le Livre de Poche, « La Pochotèque ».

Bettelheim, Bruno, (1976). Psychanalyse des contes de fées. Paris : Poche, « Pocket ».

BOCCACE, Décaméron, (2006). traduit de l'italien par Giovani Clerico. Paris : Gallimard, « Folio ».

BouloumIÉ, Arlette, (1988). Michel Tournier, Le roman mythologique. Paris : Corti.

DE NAVARRE, Marguerite, (1559). L'Heptaméron, Paris.

ECO, Umberto, Lector in fabula, (1979). Paris : éditions Grasset et Fasquelle.

LAGERLÖF, selma, (1996) Le Merveilleux voyage de Nils Holgersson à travers la Suède, traduit par Marc

de Gouvenain et Lena Grumbach, Arles : Actes Sud, « Lettres scandinaves ».

JANKÉLÉVITCH, Vladimir, (1936). Paris : Flammarion, « Champs ».

LEVI-STRAuSS, Claude, (1973). Anthropologie Structurale II, Paris, Plon.

TOURNIER, Michel (2008). Le Coq de bruyère, Paris : Gallimard, « Folio ».

(1998). Eléazar, la source ou le buisson, Paris : Gallimard, « Folio ». 
(1999). Le Médianoche amoureux, Paris : Gallimard, « Folio ».

- (1996). Le Pied de la lettre, Paris : Gallimard, « Folio ».

VRAY, Jean-Bernard, (1998). Relire Tournier, Actes du colloque international Michel Tournier, Saint-

Etienne 19-20-21 novembre 1998.

VRAY, Jean-Bernard, (1997). Michel Tournier et l'écriture seconde, Lyon : Presses Universitaires de Lyon.

\section{NOTES}

1. Sera abrégé en $E$.

2. Sera abrégé en $M A$.

\section{RÉSUMÉS}

Tout naturellement désireux de retrouver la grâce des voix de l'enfance, l'écrivain privilégie la forme du conte qu'il juge rassurante, poétique, et dont les rythmes sont envoutants. Néanmoins, il détourne, par exemple dans les Sept Contes chacune des narrations de référence : le père Noël se transforme en mère Noël et devient contemporain, pris entre les querelles des laïques et des chrétiens, le petit poucet, exilé à la ville, regrette sa vie à la campagne. L'ironie, qui superpose une voix supplémentaire, a-t-elle pour objet de mettre à distance le charme du carmen, de créer la complicité nécessaire à l'humour ou de produire un sens philosophique ? Les variations entre les différents genres, du conte bref, aux nouvelles plus graves du Médianoche amoureux conduiront sans doute à évoquer une forme de re-poétisation, bienveillante et amusée.

Spontaneously eager to imitate the charming voices of childhood, the writer most preferably choses the literary pattern of tales, which he considers both soothing and poetical and whose rhythms are bewitching. Nevertheless, he twists in his own way every narrative usual pattern, for instance in Sept Contes (Seven Tales): Santa Claus transforms into a woman of nowadays, and embodies the quarrels of Christians against the supporters of laicity. Little thumb is exiled in town where he yearns for his former country side life style. Does irony, which adds to the text an extra voice, aim at putting the charm of carmen away, or does it create a common understanding, essential to wit, or does it aim at creating a more abstract philosophical meaning?The subtle alterations from one form to another, from brief tales to the more serious short novels of Medianoche amoureux, will probably lead us to conclude to a sort of mild and playful repoetisation.

\section{INDEX}

Keywords : voices of childhood, tale, irony, re-writing, re-poetisation

Mots-clés : voix de l'enfance, conte, ironie, réécriture, re-poétisation 


\section{AUTEUR}

ANNIE URBANIK-RIZK

Classe préparatoire à l'Ens de Lyon (Académie de Créteil )

annie.rizk[at]gmail.com 